\title{
CARACTERIZACIÓN DE LA PARÁFRASIS EN ESTUDIANTES UNIVERSITARIOS
}

\author{
María Luisa Cepeda Islas, Hortensia Hickman Rodríguez, J. Jesús Becerra Ramírez, Raúl Zavaleta \\ Cepeda y David Ruiz Méndez \\ Universidad Nacional Autónoma de México \\ México
}

\begin{abstract}
RESUMEN
La paráfrasis es la habilidad que tiene una persona para reformular las ideas principales de un texto o un hablante sin distorsionarlas. En el ámbito universitario es una manera de fomentar la comprensión lectora. Hoy no existe una caracterización del tipo de paráfrasis que usan los universitarios. Objetivo: Caracterizar el tipo de paráfrasis que utilizan los universitarios. Tipo de estudio descriptivo transversal, participaron 133 estudiantes de Psicología en la Facultad de Estudios Superiores Iztacala. Para la evaluación de la paráfrasis se implementó un inventario con 20 ítems diseñado con la propuesta por Barrón-Cedeño et al. (2010). Los resultados muestran que los estudiantes utilizan con más frecuencia la tipología de cambio de orden; respecto a la complejidad, se observa una preferencia por las paráfrasis de complejidad media. No se encontraron diferencias estadísticamente significativas entre los semestres. Se sugiere el diseño de programas para fortalecer dicha habilidad.
\end{abstract}

Palabras Clave:

caracterización, paráfrasis, comprensión lectora, estudiantes universitarios, habilidades.

\section{CHARACTERIZATION OF THE PARAPHRASE IN UNIVERSITY STUDENTS}

\begin{abstract}
Paraphrase is the ability of a person to reformulate the main ideas of a text or a speaker without distorting them. In the university field it is a way to promote reading comprehension. At present there is no characterization of the type of paraphrase that university students use. Objective: To characterize the type of paraphrase that university students use. Type of cross-sectional descriptive study, 133 psychology students participated in the Faculty of Higher Education Iztacala. For the evaluation of the paraphrase an inventory with 20 items designed under the proposal by Barrón-Cedeño et al. (2010). The results show that students use the typology of order change more frequently, in terms of complexity, a preference is observed for paraphrases of medium complexity. No statistically significant differences were found between semesters. The design of programs to strengthen this ability is suggested.
\end{abstract}

\section{Keywords:}

characterization, paraphrase, reading comprehension, university students, skills.

Bitácora del Artículo:

| Recibido: 15 de Noviembre de 2019 | Aceptado: 22 de Noviembre de 2019 | Publicado en línea: Julio - Diciembre 2020 | 


\title{
Autoría y Derechos de Propiedad Intelectual
}

\section{CARACTERIZACIÓN DE LA PARÁFRASIS EN ESTUDIANTES UNIVERSITARIOS}

\author{
María Luisa Cepeda Islas, Hortensia Hickman Rodríguez, J. Jesús Becerra Ramírez, Raúl Zavaleta \\ Cepeda y David Ruiz Méndez \\ Universidad Nacional Autónoma de México \\ México
}

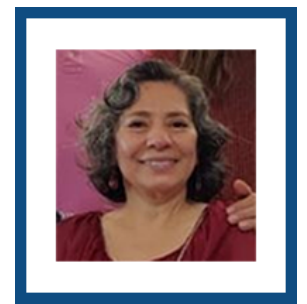

María Luisa Cepeda Islas

FES- Iztacala, UNAM

Correo: mcepedaislas@gmail.com

Profesor titular "C" T. C., Facultad de Estudios Superiores Iztacala, UNAM. Licenciada en Psicología por la Facultad de Estudios Superiores Iztacala, maestra en Modificación Conductual por la FES-I, doctora en Educación por la Universidad Anáhuac.

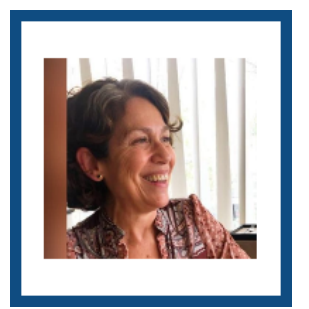

Hortencia Hickman Rodríguez

FES- Iztacala, UNAM

Correo:

Profesora titular "C", T. C., Facultad de Estudios Superiores Iztacala, UNAM. Licenciada y maestra en Psicología por la FES-Iztacala, y doctora en Pedagogía por la Facultad de Filosofía y Letras, UNAM.

\section{CONTRIBUCIÓN DE LOS AUTORES}

De manera conjunta los cinco autores desarrollamos cada etapa del artículo desde su planeación hasta la última corrección.

\section{AGRADECIMIENTOS}

Los resultados del presente artículo fueron gracias al apoyo y financiamiento del PAPIIT IT300119

\section{DATOS DE Filiación de los AUtores}

Facultad de Estudios Superiores Iztacala, UNAM

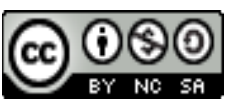

Copyright: @ 2020 Cepeda-islas, M.L., Hickman-Rodríguez, H., Becerra-Ramírez, J. J. Zavaleta-Cepeda, R. \& Ruíz-Méndez, D.

Este es un artículo de acceso abierto distribuido bajo los términos de la licencia Creative Commons Reconocimiento-NoComercial 4.0 Internacional, por lo que su contenido gráfico y escrito se puede compartir, copiar y redistribuir total o parcialmente sin necesidad de permiso expreso de sus autores con la única condición de que no se puede usar con fines directamente comerciales y los términos legales de cualquier trabajo derivado deben ser los mismos que se expresan en la presente declaración. La única condición es que se cite la fuente con referencia a la Revista Digital Internacional de Psicología y Ciencia Social y a sus autores. 


\section{TABLA DE CONTENIDO}

INTRODUCCIÓN

MÉTODO

Participantes, 307

Materiales, 307

Procedimiento, 307

Mediciones, 307

Análisis estadísticos, 308

RESULTADOS

DisCUSIÓN Y CONCLUSIONES

REFERENCIAS 


\section{INTRODUCCIÓN}

$\mathrm{E}$ ntre las diferentes problemáticas que enfrenta la educación superior en México destacan la reprobación y la discontinuidad académica. La primera se entiende como resultado de una evaluación de habilidades donde el alumno no se ha ajustado al criterio académico especificado en una asignatura (Ponce, 2004), y la segunda como la interrupción por parte del alumno del programa de formación académica con el cual está inscrito (Mares et al., 2013). Uno de los posibles factores contribuyentes a ambas problemáticas es la dificultad que presenta para los estudiantes entender los contenidos de una materia (Torres, 2011; Mares et al., 2013). Al respecto, diversos estudios indican que es la deficiencia en la capacidad de comprensión lectora lo que dificulta entender los contenidos impartidos (Irigoyen, Jiménez y Acuña, 2013). Por otra parte, se ha especulado que un nivel alto de comprensión lectora constituye un elemento clave para acceder a los contenidos, y por tanto para tener éxito académico (Hoyos y Gallego, 2017).

La investigación acerca de la comprensión lectora ofrece algunas opciones epistemológicas para el estudio del fenómeno, así como una perspectiva más clara de los factores comportamentales involucrados. Una de dichas opciones es el modelo para el análisis y evaluación de los textos científicos (Santoyo y Sánchez, 1997). El modelo intenta proporcionar una metodología de análisis y evaluación de textos científicos al alumno, estableciendo como punto central el desarrollo de habilidades metodológicas y conceptuales (HMC) (Santoyo, Colmenares y Morales, 2010). Específicamente, el modelo propone la identificación y segmentación del análisis de un texto científico partiendo de la identificación y análisis de 10 componentes, a saber: 1) justificación del estudio; 2) supuestos básicos de la investigación; 3) objetivo o propósito del estudio; 4) unidad de análisis propia del estudio; 5) estrategia del autor para el desarrollo del estudio; 6) consistencia interna del estudio; 7) consistencia externa del estudio; 8) conclusiones del autor; 9) conclusión personal del lector, y 10) cursos de acción alternativos (derivación de propuestas novedosas para el desarrollo de otras investigaciones). Hay evidencia que indica que el uso del modelo fortalece en los lectores habilidades benéficas para la formación universitaria, como la recopilación y organización de información de manera coherente (por medio de los componentes 1 a 5), la elaboración de un análisis crítico de la información (componentes 6 a 9) y la solución de problemas y toma de decisiones de acuerdo con lo leído (componente 10) (Jiménez, Santoyo y Colmenares, 2014; Moreno, Zambrano, García, Cepeda, Peñalosa y Coronado, 2008). Una serie de estudios que parten del uso y entrenamiento de alumnos en este modelo han generado evidencia de sus beneficios en la comprensión lectora en alumnos de primer semestre de la carrera de Psicología, quinto semestre de Biología y alumnos de maestría (Cepeda, Santoyo y López, 2007; Cepeda, Santoyo, Plascencia y López, 2008; Cepeda, Santoyo y López, 2009).

Los estudios citados tuvieron el objetivo común de entrenar a los alumnos en el manejo y uso de la estrategia de análisis de textos, pero con diferentes poblaciones. Se usó una metodología similar tanto en la selección de los participantes como en el diseño de investigación. En la selección de los participantes se utilizó un muestreo accidental o intencional no probabilístico. En cuanto al diseño de investigación, el que se utilizó fue el diseño cuasiexperimental Pretest y Postest. En todos los casos, tanto en el prestest como en el postest, los grupos se evaluaron de acuerdo con las categorías del modelo por medio de un cuestionario y posterior a la lectura de un texto científico, cuyas características respecto a extensión, temática, idioma (español) fueron semejantes.

Una vez hecho el pretestet, se desarrolló la intervención conformada por tres fases. En la primera se dio a conocer la estrategia y luego se aplicó con ayuda del profesor. La segunda fase tuvo como objetivo comprender los conceptos propios de una temática disciplinar propia del programa de estudios vigente en ese momento. Y la tercera para que los estudiantes por sí mismos utilizaran la estrategia en el análisis de investigaciones publicadas en revistas científicas de acuerdo con los temas revisados en clase. El análisis de los datos se efectuó por medio de tipificar las respuestas a los cuestionarios, en función del índice de aciertos.

A pesar de los resultados positivos, se observó que los niveles de ejecución de los alumnos nunca llegaron a superar $80 \%$ del dominio de los 10 componentes del modelo. ¿Qué variables están involucradas en este resultado? Algunas de las posibles respuestas se hallan en el nivel de conocimientos metodológicos, las habilidades verbales de cada alumno y los hábitos de lectura (Bazán, García y Borbón, 2005; Cepeda y López, 2010; Zarzosa, 2016). Dentro de las habilidades verbales pertinentes a la comprensión de textos científicos está la paráfrasis (Díaz-Barriga y Hernández, 2002). Al respecto varios autores (Comedarín y Medina, 1995; Fernández, 2010; Angoa, 2013) han propuesto a la paráfrasis como una vía para la comprensión y el análisis de la lectura. 
En el ámbito universitario esta habilidad es importante porque es una manera de prevenir el plagio.

Por su parte, Molina, Ríos y Rivera (2018) establecen que la enseñanza de la estrategia cognitiva como la paráfrasis textual optimiza los procesos comprensivos, y además su uso en contextos específicos. En este estudio se propone a la paráfrasis de tipo cognitivo y se vale de los saberes lingüístico del educando, en cuanto al uso de sinónimos, antónimos y otros fenómenos parafrásticos, los cuales se articulan con los conocimientos del mundo.

De manera general, el Diccionario de terminología lingüística actual define la paráfrasis como "sinonimia entre oraciones" y como "una operación en la que un enunciado primitivo o parte de él es remplazado por otro enunciado o parte de él, conservando el significado primitivo" (Garvin y cols, 1967, citado en Werner, 1981 p. 345). Romo (2007) plantea que la paráfrasis "es una operación intelectual que consiste en trasladar con nuestras propias palabras las ideas que ha expresado de manera oral o escrita otra persona, con el fin de sustituir la información a un lenguaje más personalizado y lograr una mejor comprensión" (p. 1). Martín-Antón, Carbonero y Román (2012), afirman que la paráfrasis consiste en comunicar de manera opcional una información respecto a otra ya presentada. Por su parte Barrón-Cedeño, Vila y Rosso (2010) definen las paráfrasis como las expresiones lingüísticas diferentes de la forma, pero con casi el mismo significado. En síntesis, se puede establecer que la paráfrasis es una habilidad que tiene el lector de reformular las ideas principales de un texto sin distorsionarlas, requiriendo un dominio del idioma para poder comprender el significado del autor. Supone reproducir los argumentos del emisor utilizando vocabulario y sintaxis propia para trasladar la información obtenida a las propias palabras de quien parafrasea. Esto requiere de cierta habilidad verbal en cuanto al manejo de sinónimos y antónimos, entro otros elementos léxicos.

Se ha mencionado que la habilidad de paráfrasis en estudiantes universitarios es una vía para la comprensión y el análisis de la lectura, facilitando la adquisición del conocimiento por parte de los alumnos (Comedarín y Medina, 1995; Fernández, 2010; Angoa, 2013). La investigación acerca de la temática de paráfrasis se ha caracterizado por desarrollarse con cuatro perspectivas: 1) como estrategia para el aprendizaje; 2) como medio de evaluación para la comprensión lectora; 3) como procedimiento para la eliminación de información redundante, y 4) como manera de entrenamiento. Dentro de la investigación de la paráfrasis como estrategia de aprendizaje se le ubica en el proceso de codificación, y es útil para evitar el aprendizaje repetitivo y facilitar un aprendizaje significativo (Martín-Antón, Marugán, Catalina y Carbonero, 2013). Román (2004) la plantea como estrategia de estudio para que los alumnos desarroIlen investigaciones documentales evitando el plagio. A su vez indica que la paráfrasis es un paso inicial para la comprensión de textos, lo cual conduce al análisis, formación de conclusiones y a la redacción de documentos escritos. Se parte de los conocimientos previos, apoyándose en un vocabulario (léxico) y organizaciones gramaticales aprendidas en experiencias, así como la tarea establecida y la estructura del texto (Werner, 1981; McNamara, Levinstein y Boonthum, 2004).

La paráfrasis es de uso cotidiano en la forma de lenguaje oral, por lo que con frecuencia se asume como una habilidad común en el estudiante universitario (Martín-Antón, 2008). Sin embargo, debido a los diversos modos de elaborar una paráfrasis, esta habilidad puede presentarse en distintos niveles de dominio en un alumno. Dichos niveles de dominio se pueden reflejar en gran diversidad de expresiones lingüísticas porque al hacer una paráfrasis esta puede involucrar morfología, léxico sintáctico, semántico y pragmático, concibiendo así a la paráfrasis como un fenómeno complejo (Barrón-Cedeño, Vila y Rosso, 2010). Son estos elementos los que se pueden observar en la paráfrasis, que han estipulado diversos sistemas de tipologías de paráfrasis. Cada tipo de paráfrasis está definido a partir de elementos lingüísticos y de estrategias discursivas. De esta manera, con el uso de este sistema de clasificación es posible el estudio de la paráfrasis en estudiantes universitarios y la elaboración de estrategias de entrenamiento. Barrón-Cedeño, Vila y Rosso (2010) proponen la agrupación de la paráfrasis en cinco grandes grupos, contando a su vez cada grupo con diferentes modalidades.

1. Sustitución. Remplazo de una pieza léxica por otra.

1.1. Sinonimia. Sustitución de una pieza léxica por un sinónimo.

1.2. Antonimia. Sustitución de una pieza léxica por su antónimo. Dicha sustitución se acompaña de otro tipo de modificaciones.

1.3. Generalización/Especificación. Sustitución de una pieza léxica por otra de contenido más genérico, o viceversa.

1.4. Sustitución acción-actante. Sustitución de una pieza léxica que representa la acción por otra que representa uno de los actantes de dicha acción, o viceversa.

1.5. Sustitución palabra-definición. Sustitución de una pieza léxica por su definición, o viceversa.

2. Eliminación. Supresión de una o más piezas léxicas.

2.1. Eliminación de contenido no preposicional. Supresión de una o más piezas léxicas de contenido no preposicional. 
2.2. Eliminación de argumentos. Quitar una o más piezas léxicas que representan uno de los argumentos de predicado.

2.3. Eliminación de adjuntos. Excluir una o más piezas léxicas que constituyen adjuntos del predicado.

3. Cambio en la estructura argumental. Cambio en el tipo de argumento regido por el verbo.

4. Transformación. Transmutar la estructura oracional o sintagmática.

4.1. Segmentación. Dividir la estructura oracional o sintagmática en dos o más estructuras independientes.

5. Cambio de orden. Mudar de orden en las piezas léxicas.

Con esta clasificación es posible identificar diversos niveles de complejidad de la elaboración de una paráfrasis, posibilitando así la evaluación del grado de dominio de esta habilidad en estudiantes universitarios. Como se ha indicado, dentro de las habilidades verbales que el alumno debe tener para la comprensión de textos durante su formación universitaria, la paráfrasis adquiere gran importancia (Martín-Antón, 2008).

La importancia de estudiar la paráfrasis se relaciona con los métodos de alto nivel de la composición escrita (Martín-Antón, Román y Carbonero, 2005), además que impide el aprendizaje repetitivo (Pozo, 1999). Sin embargo, el uso de la paráfrasis debe ser dirigida por el profesor, además de tenerlo en cuenta en sus procedimientos de evaluación. Por lo anterior, es necesario reflexionar acerca de qué es y cómo se presenta la paráfrasis en los alumnos universitarios para poder orientar el desarrollo de vías opcionales en la enseñanza. Para ello es necesario en primera instancia observar los niveles de elaboración de la paráfrasis para impactar de manera directa los procesos de enseñanza. Debido a esto, el objetivo del presente trabajo es caracterizar el tipo de paráfrasis utilizado por los estudiantes universitarios, partiendo del sistema de clasificación propuesto por Barron-Cedeño, Vila y Rosso (2010), así como evaluar si hay algún cambio en tipo de paráfrasis utilizada al progresar la formación del alumno.

\section{MÉtodo}

\section{Participantes}

En la Facultad de Estudios Superiores Iztacala se solicitó permiso especial en la carrera de Psicología para hacer un muestreo aleatorio estratificado de alumnos de Psicología. Como criterio de inclusión se verificó que los alumnos seleccionados estuvieran estudiando en uno de los semestres de la carrera. El estrato utilizado fue el semestre de pertenencia (primero, tercero y quinto semestres). Se obtuvo un total de 133 estudiantes con un nivel de edad de 17 y 26 años $(M=19.71, D E=1.59)$.

\section{Materiales}

Se utilizó el cuestionario de parafraseo elaborado exprofeso para el estudio (Romero, Zavaleta, Ramírez, Hickman y Cepeda, 2017). Dicho cuestionario contiene 20 ítems y una hoja de respuestas. Cada ítem consiste en la presentación de un enunciado base, el cual presenta la información "original" de la cual parte el alumno para elaborar la paráfrasis. Se presentan tres opciones con diferentes frases elaboradas a partir del enunciado original. Cada opción representa un tipo de paráfrasis de acuerdo con la propuesta de Barrón-Cedeño, Vila y Rosso (2010). Cada enunciado se elaboró a partir de los lineamientos establecidos por Coon (2005).

\section{Procedimiento}

Para este estudio se utilizó un diseño descriptivo transversal (Kerlinger, 2002). De acuerdo con lo anterior, se hizo una sola medición en el tiempo buscando evaluar las diferencias en frecuencia relativa del tipo de paráfrasis utilizada en un semestre y las variaciones en la frecuencia relativa de la paráfrasis al comparar entre distintos semestres. Se contactó a los estudiantes para solicitar su participación voluntaria en el estudio. Al aceptar participar se les citó en un salón de clases para contestar el cuestionario. Cuando los alumnos Ilegaron al aula se les solicitó leer y firmar un consentimiento informado donde se explicaban las características del estudio y se garantizaba la privacidad de los datos. Una vez firmado el consentimiento, se les colocó en un pupitre a cada uno y se les indicó que leyeran las instrucciones acerca del Ilenado del cuestionario. En seguida se atendieron dudas relacionadas con el Ilenado. Cuando los estudiantes no manifestaron tener más dudas se les indicó que iniciaran la actividad. Al finalizar se recolectaron los cuestionarios contestados y se agradeció a los participantes.

\section{Mediciones}

Para facilitar la comprensión del funcionamiento del instrumento y los diferentes tipos de paráfrasis utilizados, a continuación se presenta una serie de ejemplos que muestran una oración original y el tipo de paráfrasis asociada. En la tabla 1 se muestra el tipo de paráfrasis más básico, el cual implica el Cambio de orden. 
Tabla 1. Cambio de orden

La paráfrasis es una habilidad del lector de Idea original reformular las ideas principales de un texto sin distorsionarlas comprendiendo el significado.

Habilidad del lector de reformular las ideas

Paráfrasis principales de un texto sin distorsionarlas comprendiendo el significado.

Por otro lado, en la tabla 2 se muestra un ejemplo de la categoría de Eliminación de contenido no proposicional, la cual se caracteriza por la eliminación de una o más piezas léxicas de contenido no proposicional.

Tabla 2. Eliminación de contenido no proposicional

\section{El sistema endócrino proporciona} comunicación química en el cuerpo por medio Idea original de la liberación de hormonas en el torrente sanguíneo. Las glándulas endócrinas influyen los estados de ánimo, el comportamiento e incluso la personalidad.

El sistema endócrino proporciona

Paráfrasis comunicación química liberando hormonas en el torrente sanguíneo. Las glándulas endócrinas afectan los estados de ánimo.

En la tabla 3 se muestra un ejemplo de la paráfrasis de Eliminación de adjuntos, la cual se refiere a la eliminación de una o más piezas léxicas que constituyen elementos adjuntos del predicado.

Tabla 3.

\section{Eliminación de adjuntos}

En el condicionamiento clásico, un estímulo Idea original que antes era neutral comienza a producir una respuesta por medio de la asociación con otro estímulo.

En el condicionamiento clásico, un estímulo Paráfrasis comienza a producir una respuesta por medio de la asociación.

La tabla 4 muestra un ejemplo de la paráfrasis de Eliminación de argumentos. En esta categoría se eliminan una o más piezas léxicas que representan uno de los argumentos del predicado.

Tabla 4.

Eliminación de argumentos

Buena parte del aprendizaje humano se logra por medio de la observación o modelamiento. Las Idea original características personales del modelo influyen en el aprendizaje por observación. Los estudios han demostrado que la agresión se aprende y se libera con facilidad por medio del modelamiento.
El aprendizaje humano se logra por medio de la observación. Las características personales Paráfrasis del modelo influyen en el aprendizaje por observación. Los estudios han demostrado que la agresión se aprende y se libera con facilidad.

Por último, en la tabla 5 se muestra un ejemplo de la categoría Transformación de la estructura oracional o sintagmática. Esta categoría se caracteriza por el paso de voz activa a pasiva.

Tabla 5.

Transformación de la estructura de la oración

La cantidad mínima de energía física necesaria para producir una sensación define Idea original el umbral absoluto. La cantidad de cambio necesaria para producir una diferencia apenas perceptible en un estímulo define a un umbral diferencial.

Existen dos tipos de umbrales; en el absoluto se requiere una pequeña cantidad de energía

Paráfrasis física para producir una sensación, y en el diferencial es necesaria una determinada cantidad para producir una diferencia.

\section{Análisis estadísticos}

El análisis de datos se efectuó de dos maneras, una descriptiva en la cual se presentan las frecuencias de las elecciones hechas por los alumnos del tipo de paráfrasis, y la otra de tipo estadístico, en el cual se elaboró prueba chi cuadrada para una muestra para evaluar la distribución de las frecuencias por semestre. Los datos reunidos fueron recuperados de la hoja de respuestas y fueron procesados y analizados con Excel 2013 y SPSS versión 22.

El examen de datos se enfocó en el análisis de la distribución relativa de elecciones de paráfrasis de acuerdo con los ítems del inventario; se realizó para cada semestre y después se hizo una comparación estocástica entre las distribuciones relativas de elección de paráfrasis entre los diferentes semestres.

\section{Resultados}

Entre los principales resultados encontrados, en la tabla 6 se muestra la distribución de elecciones de paráfrasis en frecuencias absolutas y relativas para cada semestre. En dicha tabla se observa un patrón general. Para los tres semestres, los alumnos seleccionaron con más frecuencia la paráfrasis de cambio de orden (tabla 1). El segundo tipo de paráfrasis más utilizado fue el de eliminación no proposicional (tabla 2). Ambos valores de frecuencia absoluta y relativa se indican con negritas en la tabla. 
Un aspecto para destacar es que la elección a la categoría de paráfrasis predominante en primero y segundo semestre (cambio de orden) tiene un aumento sustancial para los alumnos de quinto semestre. Por otro lado, para todos los semestres la paráfrasis con menor índice de elección fue segmentación, seguida por acción actuante y generalización. Para verificar la distribución de elección de paráfrasis en cada grupo correspondiente a un semestre, se utilizó una prueba chi cuadrada para una muestra de alumnos en cada semestre. El propósito de este análisis fue evaluar si la distribución de índices en cada tipo de paráfrasis era equitativa o si había una desviación sistemática hacia un tipo de paráfrasis específica. El valor de probabilidad se ajustó de acuerdo con tres comparaciones con la corrección Bonferroni. Para primer semestre $\chi(9)=518.92, p=0.00001$; para tercer semestre $\chi(9)=238.381, p=0.000001$, y quinto semestre $\chi(9)=2073.779, p=0.0000001$; hay evidencia de una concentración sistemática de frecuencias hacia un tipo de paráfrasis particular. Lo anterior respalda la concentración de frecuencias de elección observadas en el tipo de paráfrasis de "cambio de orden".

Tabla 6. Frecuencias absolutas y relativas del tipo de paráfrasis escogida de acuerdo al semestre

\begin{tabular}{|c|c|c|c|c|c|c|}
\hline \multirow[b]{2}{*}{$\begin{array}{l}\text { TIPO DE } \\
\text { PARÁFRASIS }\end{array}$} & \multicolumn{3}{|c|}{$\begin{array}{l}\text { FRECUENCIAS } \\
\text { ABSOLUTAS }\end{array}$} & \multicolumn{3}{|c|}{$\begin{array}{c}\text { FreCuENCIAS RELATIVAS } \\
(\%)\end{array}$} \\
\hline & 舀 & 总 & 을 & 舀 & 缹 & 을 \\
\hline $\begin{array}{l}\text { Cambio de } \\
\text { orden }\end{array}$ & 248 & 130 & 566 & 29.52 & 25.79 & 49.56 \\
\hline Sinonimia & 32 & 25 & 38 & 3.81 & 4.96 & 3.33 \\
\hline Antonimia & 32 & 48 & 36 & 3.81 & 9.52 & 3.15 \\
\hline Generalización & 52 & 20 & 50 & 6.19 & 3.97 & 4.38 \\
\hline Acción actuante & 46 & 21 & 77 & 5.48 & 4.17 & 6.74 \\
\hline ENP & 146 & 89 & 136 & 17.38 & 17.66 & 11.91 \\
\hline Argumento & 100 & 47 & 80 & 11.90 & 9.33 & 7.01 \\
\hline Adjuntos & 89 & 66 & 86 & 10.60 & 13.10 & 7.53 \\
\hline Transformación & 78 & 46 & 56 & 9.29 & 9.13 & 4.90 \\
\hline Segmentación & 17 & 1 & 17 & 2.02 & 2.38 & 1.49 \\
\hline
\end{tabular}

Para dar seguimiento a la diferencia observada en el aumento de las frecuencias de paráfrasis de cambio de orden en quinto semestre, se hizo la prueba chi cuadrada con el cálculo exacto de Fisher relacionando la categoría tipo de paráfrasis con semestre de procedencia. Los resultados muestran la evidencia de diferencias entre la distribución de frecuencias de elección de paráfrasis por semestre $\chi=174.84, p<0.0001$.

\section{DisCUSIÓN Y CONCLUSIONES}

La paráfrasis como habilidad se ha utilizado como estrategia de aprendizaje, como un paso previo a la comprensión lectora y, en el ámbito universitario, esta habilidad es importante porque es una manera de prevenir el plagio. Diversas investigaciones la han relacionado con procesos de alto nivel de composición escrita (Wong, 1998).

La paráfrasis puede ser un fenómeno complejo si se distinguen las diferentes maneras (tipología) por lo que el objetivo del trabajo fue evaluar o diagnosticar el tipo de paráfrasis que muestran los alumnos de la carrera de Psicología en la FES Iztacala; este esfuerzo, aunque no único, forma parte del interés por presentar opciones de solución para alumnos universitarios en comprensión lectora.

Los resultados indican que la paráfrasis seleccionada con más frecuencia por los estudiantes corresponde a cambio de orden; a su vez, la tipología seleccionada con menor frecuencia es la antonimia. Estos datos son consecuentes con los obtenidos en el estudio de Romero, Zavaleta, Ramírez, Hickman y Cepeda (2017); sin embargo, en este estudio se observó que la tipología seleccionada con más frecuencia que la reportada en los resultados fue la de transformación, considerada con mayor complejidad.

En la literatura de la lingüística se han construido varias tipologías de paráfrasis, como las de Dras (1999), Fujita (2005) y Bhagat (2009), entre otras; en dichas clasificaciones se observa que abordan la paráfrasis como un fenómeno complejo porque esta se puede mostrar con una amplia variedad de manifestaciones de límites difusos, además que puede implicar conocimientos gramaticales de tipo morfológico, léxico, sintáctico, semántico y pragmático. Sin embargo, las categorías que se utilizaron para analizar los datos del presente estudio pretenden ofrecer una visión amplia e inclusiva del fenómeno de la paráfrasis, sin enfocarse en los mecanismos morfológicos, léxicos o sintácticos concretos. El estudio se hizo en función de la operación que desarrollan los alumnos para la identificación de los tipos de paráfrasis. De este modo se puede interpretar que los alumnos identificaron los tipos más elementales de paráfrasis, ya que por un lado se observó la categoría de cambio de orden y por otro la de eliminación de elementos. En ambas categorías sólo se hizo una modificación en la estructura de la oración, y no se evidencia un cambio a nivel gramatical; por lo tanto, 
la operación efectuada por los alumnos fue de tipo más perceptual que conceptual.

Otro dato importante en los resultados es que la prevalencia de paráfrasis más sencillas no se afecta por el semestre de procedencia, lo que podemos explicar en el sentido de que para parafrasear es necesario del dominio del idioma para comprender el significado del autor (Arechabala, Catoni, Ávila, Riquelme y Aedo, 2011; Arbusti, 2010; León, Solari y Escudero, 2011), lo que implicaría que el nivel lingüístico de los estudiantes es bajo. Por su parte, McGuire y Yewchuk (1996) indican que la presencia de la paráfrasis está asociada a sujetos de altas capacidades, y que además evitan el aprendizaje repetitivo.

Por otro lado, se observó que conforme avanza el semestre hay una concentración mayor en la paráfrasis más sencilla (cambio de orden), segmentación, generalización y acción actuante, lo cual nos permite suponer que esta habilidad no cambia al menos en los alumnos de Psicología.

Considerando los datos obtenidos, se puede suponer que los tipos de paráfrasis seleccionados con mayor índice se ubican en un nivel de complejidad elemental (cambio y eliminación), porque no implica un manejo lingüístico sino que sólo son cambios a nivel morfológico, lo que implicaría que estas habilidades dificultan a los alumnos lograr la comprensión y mucho menos la inferencia, sobre todo si se considera que la paráfrasis es un paso previo a la comprensión y a la inferencia.

En este mismo sentido, y de acuerdo con Cepeda, López y Santoyo (2013), la imposibilidad de identificar paráfrasis de mayor complejidad tal vez se deba a la escasez en el léxico de los alumnos, debido que este factor es decisivo para trasladar el texto con palabras propias del alumno. Por otro lado, uno de los factores importantes en la explicación de los resultados es el repertorio del vocabulario, porque es un insumo necesario para la reconstrucción del texto. Por otro lado, y de acuerdo con los resultados de Molina, Ríos y Rivera (2018), la identificación de paráfrasis "simples" de los alumnos sirve como indicador de la dificultad en la comprensión, porque cuando el lector no está en capacidad de expresar con otras palabras lo leído, es incapaz.

En otro estudio, Cepeda, López y Santoyo (2013) también evaluaron los tipos de parafraseo que presentaba una muestra de estudiantes universitarios, utilizando la clasificación elaborada por Fernández (2010). En la tipología utilizada se plantearon las categorías de copia textual, paráfrasis sinonímica (cambio de algunas palabras por sinónimos), paráfrasis sintáctica (cambio en el orden o estructura), combinación de las anteriores, reestructuración total (cambio tanto en la sintaxis como en la estructura sin cambiar la idea original) y la inferencia (indicar aspectos no presentes en el texto). Los datos analizados evidencian que $53 \%$ de los alumnos usó la copia textual, 1\% la paráfrasis sinonímica, 23\% la paráfrasis sintáctica, 6\% la paráfrasis sinonímica-sintáctica, 4\% la paráfrasis de restructuración total, y $13 \%$ con inferencia. En esta investigación los alumnos tenían que elaborar la paráfrasis, por lo que podemos inferir que para ellos es más difícil la elaboración que la identificación.

Es necesario considerar con reserva los resultados del presente estudio, en tanto que se deben hacer más evaluaciones, además de tener en cuenta a alumnos de otras carreras o evaluar habilidades verbales que presentan los alumnos. Otro aspecto a considerar para efectuar futuras evaluaciones es que en este trabajo se solicitó a los alumnos la identificación de los diferentes tipos de paráfrasis, y otra forma sería pedir la elaboración de paráfrasis a partir de textos. Además, los textos podrían ser clasificados por su extensión, es decir, son necesarias diferentes operaciones cuando se parafrasea una frase, un párrafo o un texto completo. Esta clase de evaluación requiere de mayor análisis en tanto que sería necesario tener categorías para clasificar las diversas respuestas de los alumnos. Por ello, consideramos que se deben establecer nuevas evaluaciones que permitan desarrollar estrategias más sistemáticas para el entrenamiento de manera explícita como estrategia de aprendizaje (McNamara, Levinstein y Boonthum, 2004).

Es necesario desarrollar programas remediales para perfeccionar la paráfrasis, y las tipologías de la paráfrasis ayudan a distinguir los elementos característicos que abrigan al fenómeno. Al respecto, la tipología presentada por Barrón-Cedeño, Vila y Rosso (2010) hace referencia a las formas lingüísticas propiamente como consecuencia de su afán por detectar el plagio. Estas categorías son definidas a partir de elementos lingüísticos y de estrategias discursivas, las cuales pueden guiar el desarrollo de una estrategia de entrenamiento.

Consideramos de gran relevancia contribuir al desarrollo de estrategias para fortalecer -o en su caso desarrollar - los repertorios de los jóvenes que ingresan al nivel de educación superior. Son variadas las iniciativas de investigadores para resolver los problemas académicos que generan los bajos desempeños académicos en los niveles de estudios superiores; valdría la pena sistematizar estas aproximaciones para que sean demostradas sus contribuciones.

A partir de lo observado en esta investigación, se hacen algunas propuestas con el propósito de mejorar el trabajo hecho en este campo. En primer lugar, como ya se indicó, se propone el desarrollo de programas de 
entrenamiento, presenciales y computacionales, que destaquen el uso de la paráfrasis como estrategia de aprendizaje destinado al perfeccionamiento de la comprensión lectora. En segundo lugar se sugiere en futuros estudios evaluar el nivel de correlación que hay entre la complejidad de la paráfrasis y la habilidad verbal.

Se propone que para futuras investigaciones se evalúe la paráfrasis y otorgue más importancia a la elaboración de paráfrasis por encima de su selección, debido a las características de éstas.

\section{Referencias}

Angoa, J. (2013). Análisis de la paráfrasis y su relación con el análisis de textos científicos. Tesis de licenciatura en Psicología. FES Iztacala, UNAM, México.

Arbusti, M. (2010). Paráfrasis, escucha y reformulación. En V. Castel y L. Cubo (eds.), Renovación de la palabra (pp. 149154). Argentina: FFyL.

Arechabala, M., Catoni, M., Ávila, N., Riquelme, G. \& Aedo, V. (2011). Géneros discursivos y errores más frecuentes en los informes académicos de estudiantes de Enfermería. Invest. Educ. Enferm., 29(3), 400-406.

Barrón-Cedeño, A., Vila, M. \& Rosso, P. (2010). Detección automática de plagio: De la copia exacta a la paráfrasis. En E. Garayzábal, M. Jiménez y M Reigosa (coords.), Panorama actual de la lingüística forense en el ámbito legal y policial: Teoría y práctica. Jornadas (in)formativas de lingüística forense (pp. 76-96). Madrid: Euphonia Ediciones SL.

Bazán, A., García, I., \& Borbón, J. C. (2005). Evaluación de habilidades metodológico-conceptuales en el análisis de textos científicos: Algunos hallazgos empíricos. En C. Santoyo (comp.). Análisis y evaluación de habilidades metodológicas, conceptuales y profesionales en la formación del psicólogo (pp. 109-127). México: UNAM.

Bhagat, R. (2009). Learning Paraphrases from Text. Tesis doctoral. California: University of Southern.

Cepeda, L., López, M. R., \& Santoyo, C. (2013). Relación entre la paráfrasis y el análisis de textos. Revista Electrónica de Investigación Educativa, 15(1), 99-106. Recuperado de http:// redie.uabc.mx/vol15no1/contenido-cepedalopez.html.

Cepeda, L., \& López, R. (2010). Análisis estratégico de textos: Fundamentos teórico-metodológicos y experiencias instruccionales. México: UNAM/PAPIME.

Cepeda, L., Santoyo, C., \& López, M. del R. (2007). Estrategia de análisis de textos científicos en estudiantes de Psicología. 2o. Congreso Internacional de Innovación Educativa. Ciudad de México, México. 13 de noviembre.

Cepeda, L., Santoyo, C., Plascencia \& López, M. del R. (2008). Relación entre habilidad verbal y análisis de textos científicos. II Congreso Internacional de Psicología. La investigación en Psicología: Entre lo real y lo posible. Morelia, México. 27-31 de octubre.

Cepeda, L., Santoyo, C., \& López, M. del R. (2009). Validación de una estrategia de análisis de textos científicos en estudiantes de maestría. Congreso Internacional para la
Investigación y el Desarrollo Educativo. Veracruz, México. Comedarín, M., \& Medina, A. (1995). Desarrollo de las competencias lingüísticas y comunicativas de los alumnos de 50. a 80. año de EGB: Fundamentos y estrategias. Chile: Ministerio de Educación.

Coon, D. (2005) Fundamentos de Psicología. México: Thomson.

Díaz-Barriga, A. F., \& Hernández, R. G. (2002). Estrategias docentes para un aprendizaje significativo. Una interpretación constructivista. México: Mc Graw-Hill.

Dras, M. (1999). Tree Adjoining Grammar and the Reluctant Paraphrasing of Text. Tesis doctoral. Australia: Macquarie University.

Fernández, C. (2010). Evaluación de la paráfrasis de textos científicos en estudiantes universitarios. Tesis de licenciatura en Psicología. México: FES Iztacala, UNAM.

Fujita, A. (2005). Automatic Generation of Syntactically WellFormed and Semantically Appropriate Paraphrases. Tesis doctoral. Japón: Nara Institute of Science and Technology.

Hoyos, A., \& Gallego, T. (2017). Desarrollo de habilidades de comprensión lectora en niños y niñas de la básica primaria. Revista Virtual Universidad Católica del Norte, 51, 23-45. Recuperado de http://revistavirtual.ucn.edu. co/index.php/RevistaUCN/article/view/841/1359.

Irigoyen, J. J., Jiménez, M., \& Acuña, K. F. (2013). Formación por competencias: Algo más que discursos. En A. Bazan y D. Castellanos. La Psicología en la educación. Contextos de aprendizaje e investigación (33-54). México: Plaza y Valdés.

Jiménez, P. A., Santoyo, V. C., \& Colmenares, V. L. (2014). El significado de la complejidad en el estudio de las habilidades metodológicas y conceptuales. En M. L. Cepeda y M. R. López (coords.). Desarrollo de habilidades complejas en la lecto-escritura y del aprendizaje científico. México: UNAM/ PAPIIT IN302110.

Kerlinger, F. (2002). Investigación del comportamiento. México: Mc Graw-Hill Interamericana.

León, J., Solari, M., \& Escudero, I. (2011). La generación de inferencias dentro de un contexto social. Un análisis de la comprensión lectora a través de protocolos verbales y una tarea de resumen oral. Revista de Investigación Educativa, 29(1), 13-42.

Mares, G., González, L., Rivas, O., Rocha, H., Rueda, E., Rojas, L., Cruz, D., y López, R. (2013). Trayectorias discontinuas en educación superior: El caso de alumnos de la carrera de Psicología de Iztacala, México. Revista Mexicana de Investigación en Psicología, 5(1). pp. 71-81.

Martín-Antón, L. (2008). Evaluación y entrenamiento en elaboración de paráfrasis y búsqueda de aplicaciones. En J. González-Pienda y J. Núñez (coords.). Psicología y educación: Un lugar de encuentro (pp. 864-869). Oviedo: Universidad de Oviedo.

Martín-Antón, L., Carbonero, M., \& Román, J. (2012). Efecto modulador de variables socioemocionales en el entrenamiento de estrategias de elaboración en educación secundaria obligatoria (ESO): Paráfrasis y aplicaciones. Psicothema, 24(1). pp. 35-41.

Martín-Antón, L., Marugán, M., Catalina, J., \& Carbonero, M. (2013). Estrategias de aprendizaje de elaboración. Entrenamientos y programas. Aula Abierta, 41(1), 49-62. 
Martín-Antón, L., Román S. J., \& Carbonero, M. (2005). La paráfrasis como estrategia de aprendizaje: Propuesta de intervención. International Journal of Developmental and Educational Psychology, vol. 2, núm. 1, 393-408.

McGuire, K. L., \& Yewchuck, C. R. (1996). Use of Metacognitive Reading Strategies by Gifted Learning Disabled Students: An Exploratory Study. Journal for the Education of the Gifted, 19(3), 293-314.

McNamara, D., Levinstein, I., \& Boonthum, C. (2004). iStart: Interactiva Strategy Training for Active Reading and Thinking. Behavior Research Methods, Instruments \& Computers, 36(2), 222-233.

Molina, S. R., Ríos, N. A., \& Rivera, Z. C. (2018). La paráfrasis textual como estrategia cognitiva para la comprensión lectora. Estudio realizado en los grados quinto y séptimo de tres instituciones educativas públicas de Yopal, Casanar. Tesis de Maestría. Colombia: Casanare.

Moreno, D., Zambrano, D., García, G., Cepeda, L., Peñalosa, E., \& Coronado, O. (2008). Análisis de textos científicos desde la web: Un estudio piloto. Enseñanza e Investigación en Psicología, 13(2), 387-40.

Ponce, G. V. (2004). Reprobación y fracaso en secundaria. Hacia una reforma integral. Revista de Educación y Desarrollo, 2, 59-70.

Pozo, J. (1999). Más allá del cambio conceptual: El aprendizaje de la ciencia como cambio representacional. Enseñanza de las Ciencias, 17(3), 503-512.

Román, J. M. (2004). Estrategia de lectura significativa de textos para universitarios. En A. Villa y otros (coords.). Pedagogía universitaria: Hacia un espacio de aprendizaje compartido (pp. 1121-1141). Bilbao: Editorial Mensajero-
ICE Universidad Deusto.

Romero, R. A., Zavaleta, C. R., Ramírez, F. J., Hickman, R. H., \& Cepeda, I. L. (2017). Paráfrasis y habilidad verbal en univerisitarios. Documento presentado en el XXXVI Coloquio de Investigaciòn. México: FES Iztacala, UNAM.

Romo, E. (2007). ¿Qué es una paráfrasis? Universidad Autónoma de Guadalajara. Guadalajara, Jalisco. Extraído el 5 de mayo, 2011 de http://genesis.uag.mx/hoy/parafrasis.cfm

Santoyo V. C., Colmenares V. L., \& Morales Ch. S. (2010). Una estrategia para el análisis de textos científicos con retroalimentación personalizada. En L. Cepeda y M. R. López (eds.). Análisis estratégico de textos: Fundamentos teórico-metodológicos y experiencias instruccionales (pp. 123-148). México: UNAM/PAPIME.

Santoyo, V. C., \& Sánchez,J. M. (1997). Una estrategia para evaluar habilidades científicas y profesionales: La experiencia de la Facultad de Psicología. En Comisión para el cambio curricular (eds.). Hacia el cambio curricular: Diagnóstico del curriculum actual de la Facultad de Psicología (pp. 1057-1093) México: Facultad de Psicología, UNAM.

Torres, L. (2011). Rendimiento académico, familia y equidad de género. Ciencia y Sociedad, 34(1), 46-64.

Werner, A. (1981). Diccionario de terminología lingüística actual. Madrid: Gruelos.

Wong, M. (1998). You Said It!: Listening/Speaking Strategies and Activities. Cambridge University Press.

Zarzosa, L. (2016). Software para el desarrollo de lectura estratégica en universitarios. En https://www. researchgate.net/publication/229005869 Software para el_desarrollo_de_lectura_estrategica_en_universitarios. 


\section{Meta-Análisis del Artículo}

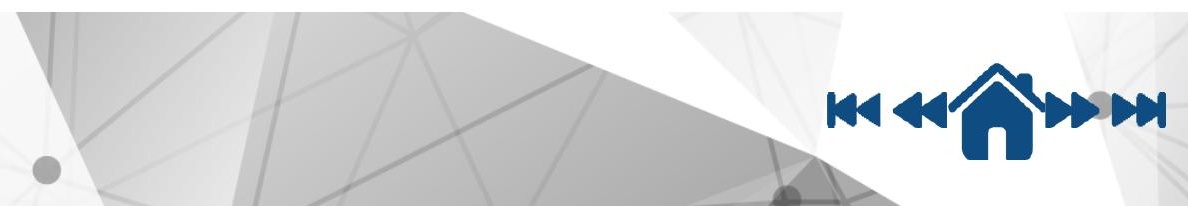


Revista Digital Internacional de Psicología y Ciencia Social |Vol. 6 | Núm. $2 \mid$ Julio-Diciembre 2020| e-ISSN 2448-8119

\section{Dimensión Cuantitativa}

\section{Perfil de Evaluación entre pares}
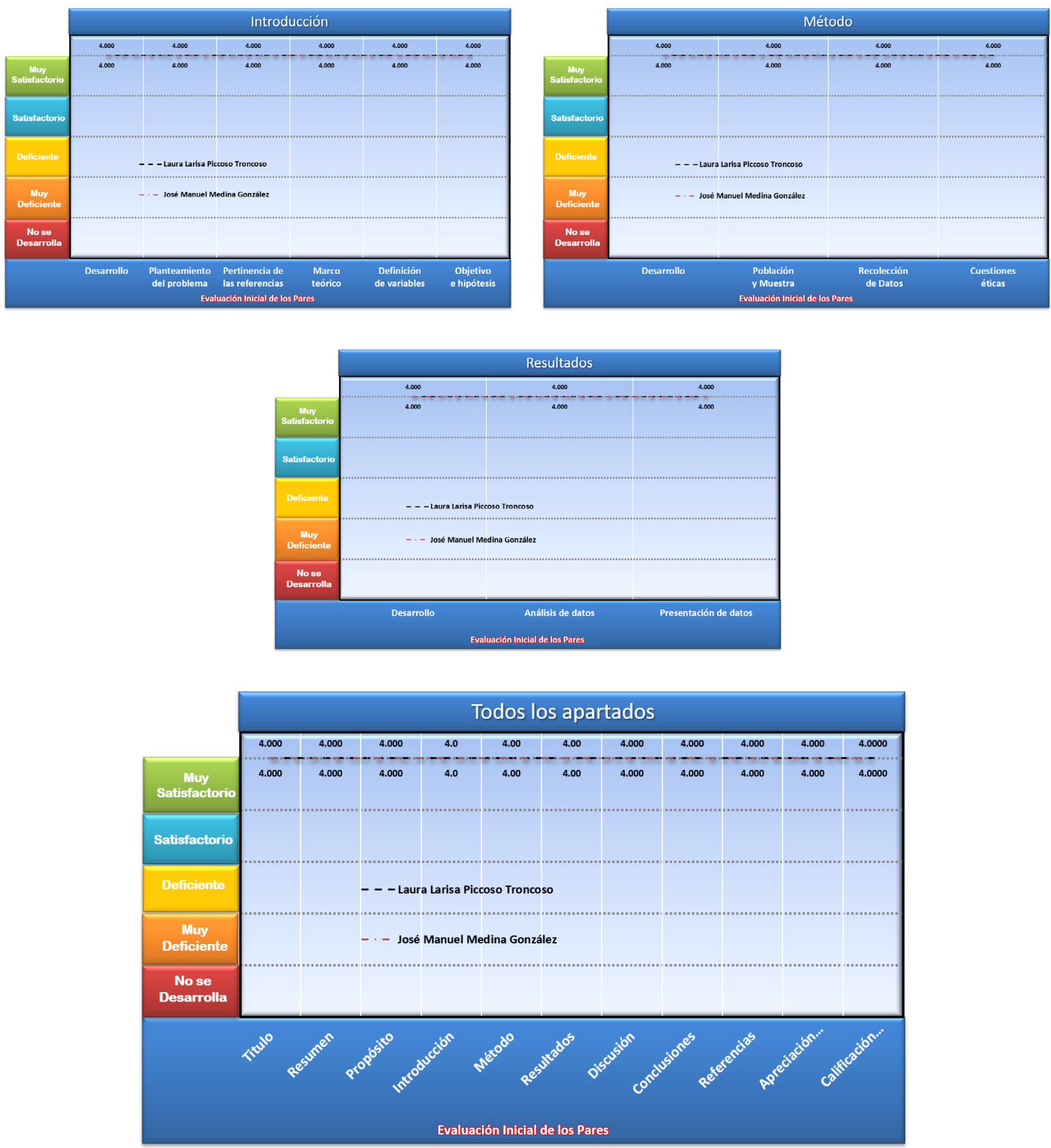


\section{Índice de Concordancia}

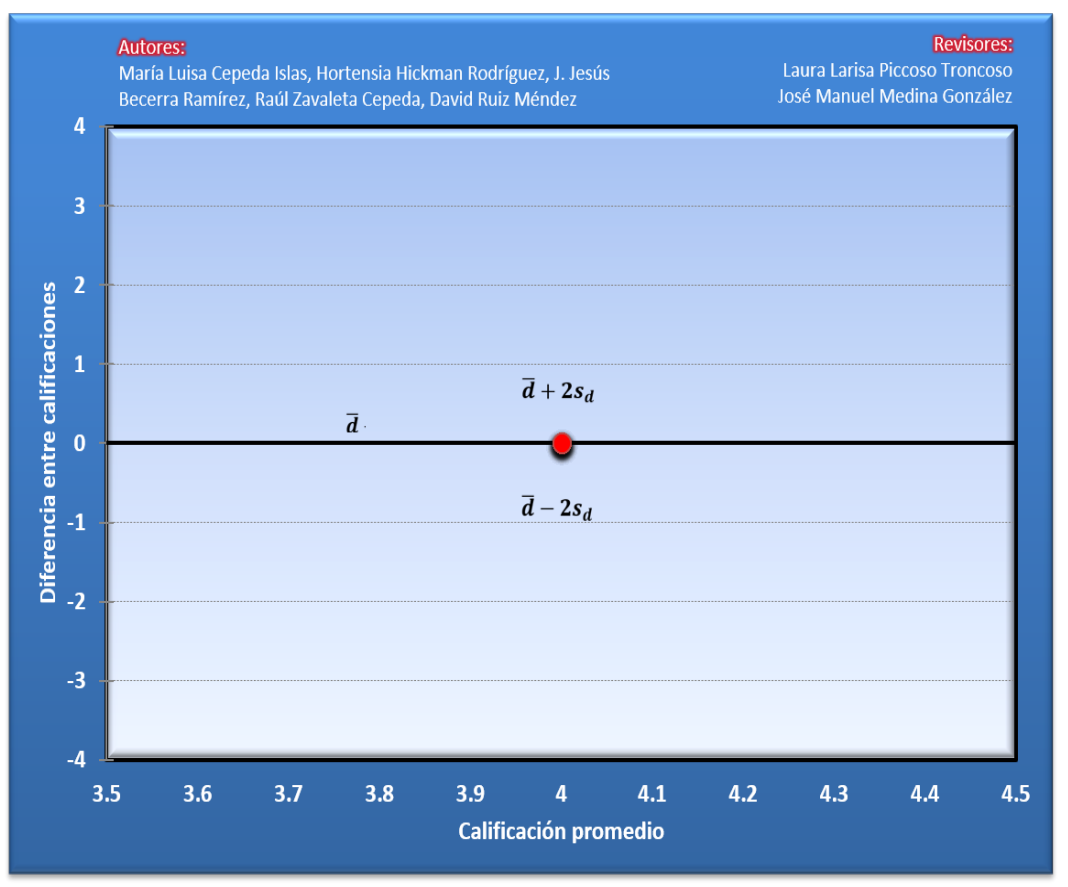

Índice de Acuerdo

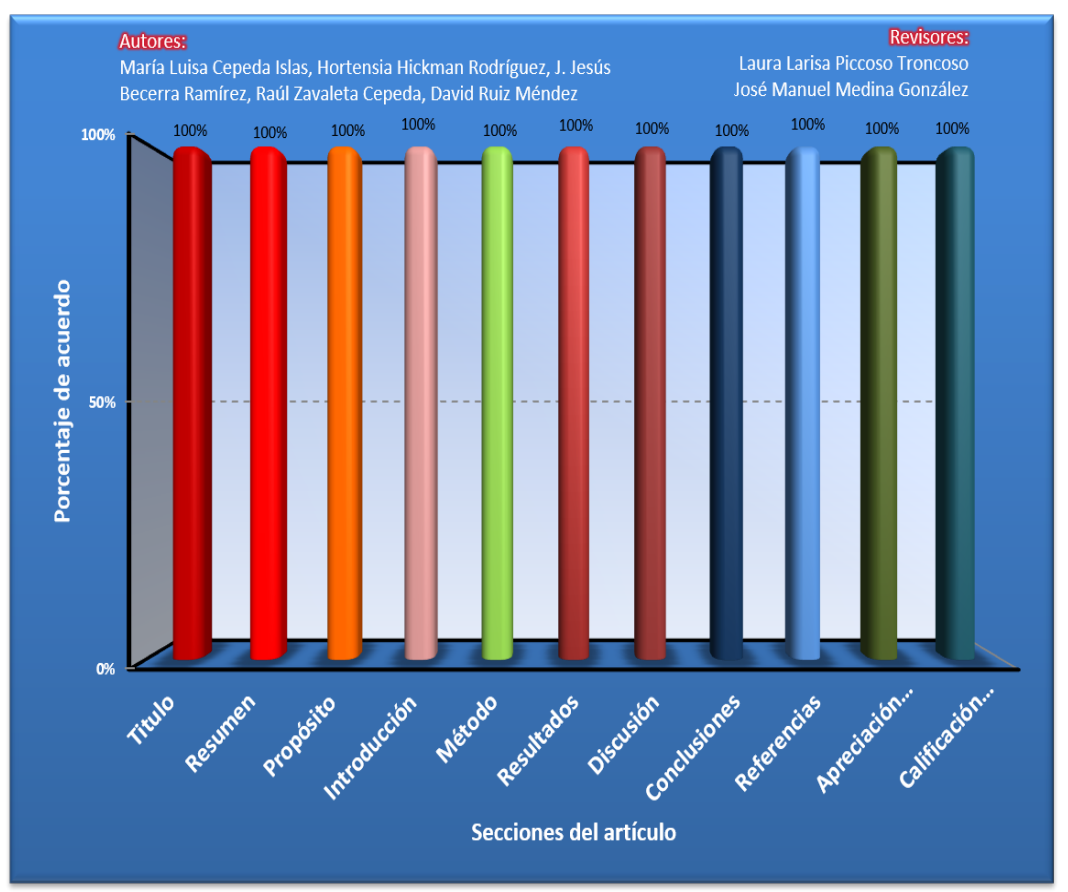




\begin{tabular}{|c|c|}
\hline Revisor 1 & Revisor 2 \\
\hline Laura Larisa Piccoso Troncoso & José Manuel Medina González \\
\hline \multicolumn{2}{|c|}{ Título/Autoría } \\
\hline El título cumple con lo requerido satisfactoriamente. & El título es correcto y cumple satisfactoriamente. \\
\hline \multicolumn{2}{|c|}{ Resumen } \\
\hline Puntual y concreto el resumen. & El Resumen es claro y satisfactorio. \\
\hline \multicolumn{2}{|c|}{ Próposito del Estudio } \\
\hline Congruente el propósito del estudio. & $\begin{array}{l}\text { El propósito del estudio da aportaciones importantes } \\
\text { correctamente detallado. }\end{array}$ \\
\hline \multicolumn{2}{|c|}{ Introducción } \\
\hline $\begin{array}{l}\text { La introducción contiene los requerimientos satisfac- } \\
\text { toriamente, permite al lector mantener el interés en la } \\
\text { lectura de la investigación. }\end{array}$ & La introducción es correcta y cumple con lo solicitado. \\
\hline \multicolumn{2}{|c|}{ Método } \\
\hline $\begin{array}{l}\text { Se verifica el proceso sistemático del trabajo en el mé- } \\
\text { todo, satisfactoriamente. }\end{array}$ & El método demuestra claridad, sistematización correcta. \\
\hline \multicolumn{2}{|c|}{ Resultados } \\
\hline $\begin{array}{l}\text { Los resultados permiten tomar decisiones importantes } \\
\text { dentro del área de investigación, son correctos, concre- } \\
\text { tos y puntuales. }\end{array}$ & $\begin{array}{l}\text { Los resultados arrojan aportaciones importantes en el } \\
\text { ámbito científico. }\end{array}$ \\
\hline \multicolumn{2}{|c|}{ Discusión } \\
\hline Buen trabajo realizado en la discusión. & La discusión es correcta y cumple con lo solicitado. \\
\hline \multicolumn{2}{|c|}{ Conclusiones } \\
\hline $\begin{array}{l}\text { Las conclusiones permiten manifestar el proceso para } \\
\text { futuras investigaciones. }\end{array}$ & Las conclusiones aportan sustento científico. \\
\hline \multicolumn{2}{|c|}{ Referencias } \\
\hline Son correctas y constan un amplio trabajo bibliográfico. & Las referencias con oportunas y adecuadas. \\
\hline
\end{tabular}




\section{Historia del Proceso EDITORIAL}

